\title{
Comparación de la fórmula de Friedewald con el método precipitado para la determinación de los niveles de colesterol LDL en bovinos
}

\author{
COMPARISON OF THE FRIEDEWALD FORMULA WITH THE PRECIPITATE METHOD FOR THE \\ DETERMINATION OF LDL CHOLESTEROL LEVELS IN CATTLE
}

José Henry Osorio ${ }^{1,2,4}$, Jorge Abel Castañeda ${ }^{3}$

\section{Resumen}

El objetivo de este estudio fue comparar dos métodos (precipitado y Friedewald) para la determinación de los valores de colesterol LDL en bovinos. Se tomaron 56 muestras de sangre de bovinos en las primeras horas de la mañana. Se determinaron los niveles de colesterol LDL en el suero mediante el método de precipitación y mediante la fórmula de Friedewald. Los resultados fueron analizados mediante un análisis de varianza de una vía. El método precipitado reportó valores $(\mathrm{mg} / \mathrm{dl})$ de 14.33, 0.36, 59.11, 58.75 y 11.21 para promedio, mínimo, máximo, rango y desviación estándar, respectivamente, y mediante el método de Friedewald se obtuvieron valores (mg/dl) de 62.04, 13.68, 156.82, 143.14 y 32.97 , respectivamente, habiendo diferencia significativa entre estos métodos para un nivel de confianza del $95.0 \%$. Los resultados indican que no es recomendable la utilización del método de precipitación para la determinación del colesterol LDL en los bovinos.

Palabras clave: colesterol LDL; bovinos; método precipitado; fórmula de Friedewald

\section{Abstract}

The aim of this study was to compare two methods (precipitate and Friedewald) for the determination of LDL cholesterol values in cattle. Blood samples were taken from 56 cattle in early morning. In the serum LDL cholesterol levels were determined by the

\footnotetext{
${ }^{1}$ Laboratorio de Investigación en Bioquímica Clínica y Patología Molecular, Departamento de Ciencias Básicas de la Salud, Universidad de Caldas, Manizales, Colombia

${ }^{2}$ Laboratorio de Investigación en Metabolismo, Universidad de Manizales, Colombia

${ }^{3}$ Facultad de Ciencias Exactas y Naturales, Universidad de Caldas, Manizales, Colombia

${ }^{4}$ E-mail: jose.osorio_o@ucaldas.edu.co
}

Recibido: 12 de mayo de 2017

Aceptado para publicación: 5 de octubre de 2017 
precipitation method and the Friedewald formula. The results were analyzed using a oneway analysis of variance. The precipitated method reported values $(\mathrm{mg} / \mathrm{dl})$ of 14.33, 0.36, $59.11,58.75$ and 11.21 for average, minimum, maximum, range and standard deviation, respectively, and by the Friedewald method values (mg/dl) were 62.04, 13.68, 156.82, 143.14 and 32.97 respectively, with significant difference between the two methods for a confidence level of $95.0 \%$. The results indicate that the use of the precipitation method for the determination of LDL cholesterol in cattle is not recommended.

Key words: LDL cholesterol; cattle; precipitation method; Friedewald formula

\section{INTRODUCCIÓN}

El colesterol y los ésteres de colesterol (EC) son lípidos importantes en la dieta de los bovinos, provienen de los fosfolípidos de las plantas y de las grasas contenidas en las dietas suplementadas (Aranda et al., 2012). En los rumiantes adultos, la digestión de lípidos se inicia en los pre-estómagos (retículo y rumen), a diferencia de los no rumiantes, donde la digestión ocurre en el intestino delgado (Bauchart, 1993). Para su transporte en el plasma, los lípidos necesitan de macromoléculas llamadas lipoproteínas (LP), ya que estos son insolubles en agua y no pueden ser trasportados en soluciones acuosas (Montgomery, 1998).

Las LP son proteínas llamadas apolipoproteínas, solubles en los fluidos de los sistemas vasculares (plasma y linfa) y en los fluidos intestinales y folicular. Su función principal es el transporte de lípidos a partir de órganos secretores (intestino e hígado) a los tejidos periféricos. El papel de la LP en la formación de micelas es mayor en los rumiantes que en los monogástricos por la ausencia de 2-monoglicéridos, los cuales constituyen un factor importante en la solubilización micelar de ácidos grasos libres (Bauchart, 1993).

Las diferencias en la densidad de flotación, el tamaño y las fracciones de LP permiten el aislamiento y la caracterización de las LP en bovinos, mediante procedimientos plan- teados para el plasma humano, como la ultracentrifugación, precipitación, electroforesis e inmunoelectroforesis. Los LP se clasifican en cinco clases de densidad que expresan su contenido lipoproteico: quilomicrones (Q), LP de muy baja densidad (VLDL), LP de baja densidad (LDL), LP de densidad intermedia (IDL) y LP de alta densidad (HDL) (Bauchart, 1993; Montgomery, 1998).

En cuanto a las LP aisladas por electroforesis, las principales clases han sido denominadas mediante un sistema análogo al utilizado para otras proteínas plasmáticas, como: alfa-lipoproteínas (HDL), betalipoproteínas (LDL) y pre-beta-lipoproteínas (IDL y VLDL) (Cirio y Tebot, 2000). El metabolismo lipoproteico revela características similares entre especies animales, pero con algunas diferencias, pues los rumiantes, así como los equinos, caninos, felinos y ratas poseen un "patrón HDL», caracterizado por el predominio de alfa-lipoproteína en el plasma (Osorio et al., 2015). Cuando estos animales son alimentados con dietas grasas, el colesterol es captado por HDL (en lugar de LDL), evitándose efectos nocivos debido a la acción protectora atribuible a las HDL. El humano, cerdos, conejos, marmotas y varias especies de monos responden al "patrón LDL», caracterizado por el aumento de betalipoproteína, y a mayor riesgo aterogénico cuando se consumen dietas excesivamente grasas (Coppo, 1992, 2001; Bauer, 1996). En animales con «patrón HDL», las dietas ricas en energía aumentarían las HDL a expensas 
de la disminución de LDL (Coppo, 1990, 2001).

La composición química y la tasa de secreción de las LP son factores que no solo controlan la utilización de lípidos, sino también las características cualitativas y cuantitativas de la producción cárnica y láctea (Osorio y Vinazco, 2010), de allí la importancia de medir los niveles de colesterol en vacas lecheras. Por otro lado, considerando que para la determinación de los niveles de LDL existen varios métodos disponibles, el presente estudio hizo una evaluación comparativa entre el método de precipitación y la fórmula establecida por Friedewald (Friedewald et al., 1972), esta última utilizada para la determinación del perfil lipídico en humanos, con el fin de validar un método confiable en las mediciones de colesterol LDL en la especie bovina.

\section{Materiales y Métodos}

Se trabajó con bovinos Bos taurus de predios bajo un sistema semi-intensivo, ubicados en el departamento de Caldas, Colombia. Los animales de los predios trabajados llevaban un adecuado plan sanitario (desparasitados y con vacunas al día), y presentaban una condición corporal entre $3 / 5 \mathrm{y}$ $4 / 5$. Se tomaron 56 muestras de sangre sin discriminación de sexo y edad.

La toma de la muestra se realizó en las primeras horas del día, antes de ser suministrado el suplemento dietario. Las muestras fueron refrigeradas para su transporte al laboratorio, donde fueron centrifugadas (centrifuga Thermo IEC CL31) a $2579 \mathrm{~g}$ durante 15 minutos. El suero resultante fue conservado a $-30{ }^{\circ} \mathrm{C}$ hasta su análisis.

Los niveles de colesterol LDL se determinaron utilizando kits comerciales (BioSystems ${ }^{\circledR}$ ) mediante el método de precipitación (estos resultados han sido previamente publicados en Osorio y Castañeda,
2018). Aquí, las LDL presentes en la muestra se precipitan en presencia de polivinil sulfato, y su concentración se calcula por diferencia entre los valores de colesterol en el suero y en el sobrenadante obtenido tras la precipitación, donde el colesterol se cuantifica espectrofotométricamente. Asimismo, se determinaron los valores LDL utilizando la fórmula establecida por Friedewald et al. (1972), donde el colesterol HDL es precipitado en presencia de ácido fosfotungstico y determinado mediante el método enzimáticocolorimétrico aplicado para la determinación del colesterol total de la muestra.

Adicionalmente se determinaron los niveles de triglicéridos (TG) mediante un método enzimático-colorimétrico (Fossati y Prencipe, 1982). Los valores de TG son divididos entre 5 y este valor corresponde a los niveles de colesterol VLDL. Los valores de colesterol LDL son calculados mediante la fórmula: Colesterol LDL $=$ Colesterol total (colesterol HDL + colesterol VLDL).

Se obtuvo estadística descriptiva para la cuantificación del colesterol LDL, tanto para el precipitado como para los valores de la fórmula de Friedewald. Las diferencias de los dos métodos se evaluaron por medio de un análisis de varianza utilizando el programa Statgraphics Plus 5.1, donde se aceptaron diferencias significativas cuando $\mathrm{p}<0.05$.

\section{Resultados y Discusión}

Los valores de colesterol LDL mediante el método precipitado y el método de Friedewald se presentan en el Cuadro 1, habiendo diferencia significativa entre ambos métodos (Cuadro 1).

En medicina veterinaria es importante evaluar el uso de métodos que tradicionalmente son utilizados en el humano. En el caso del método de Friedewald, este no utiliza mediciones de colesterol LDL, sino que los calcula mediante una fórmula (Friedewald $e t$ 
Cuadro 1. Valores de colesterol LDL (mg/dl) en bovinos Bos taurus

\begin{tabular}{lccc}
\hline Parámetros & Método precipitado $^{1}$ & $\begin{array}{l}\text { Método de } \\
\text { Friedewald }\end{array}$ & P-valor \\
\hline Promedio & 14.33 & 62.04 & \\
Desviación estándar & 11.21 & 32.97 & 0.0000 \\
Mínimo & 0.36 & 13.68 & \\
Máximo & 59.11 & 156.82 & \\
\hline
\end{tabular}

${ }^{1}$ Datos publicados en Osorio y Castañeda (2018)

al., 1972). Además, es importante que en los estudios se analice un método que permita mejor comprensión y mayor precisión sobre los procesos utilizados en los laboratorios clínicos para la cuantificación del colesterol CLDL a fin de obtener valores confiables y a bajo costo. Los métodos establecidos por la National Cholesterol Education Program (NCEP) para la determinación de C-LDL requieren de ensayos homogéneos o de ultracentrifugación, que muchas veces no son de fácil acceso para los laboratorios de rutina por su alto costo (Mendes De Cordova et al., 2004).

En humanos, el método más común para la determinación de C-LDL en el laboratorio clínico es el cálculo de Friedewald, que estima el C-LDL a partir de mediciones de CT, TG y C-HDL teniendo importancia la precisión y el rendimiento analítico. Esta fórmula fue descrita como una alternativa al método de ultracentrifugación y es el método de elección por los laboratorios clínicos de rutina (Bachorik y Ross, 1995). Sin embargo, a pesar de que la fiabilidad de las estimaciones de C-LDL disminuye considerablemente con el aumento de las concentraciones de TG (Warnick et al., 1990), el error de determinar el C-LDL a través de una estimación, suma los errores analíticos de las tres determinaciones involucradas en el cálculo, por lo que habitualmente no reúne los criterios del NCEP acerca del error total; no obstante, esta téc- nica sigue siendo válida (Mendes De Cordova et al., 2004).

Los métodos de ultracentrifugación pueden verse alterados por las concentraciones de los reactivos y las fuerzas centrífugas, dependiendo de las habilidades y la atención del técnico; sin embargo, hoy en día es considerado un método de comparación clásica y es la base para aceptar un método de referencia (Nauck et al., 2002).

En el análisis comparativo entre el método precipitado y la fórmula de Friedewald se encontraron diferencias significativas entre los valores obtenidos por ambos métodos. Este resultado se contradice con el estudio de Valverde-Chaves et al. (1994) en humanos quienes no encontraron discrepancias entre estos métodos; sin embargo, coincide con las discusiones cuando afirman que los valores alcanzados con la fórmula de Friedewald son ligeramente más altos que aquellos proporcionados por el método de precipitación.

Mendes De Cordova et al. (2004), por otro lado, concluye que el método directo es apropiado para el laboratorio de rutina y no tiene las interferencias que presentan los métodos de precipitación y los errores adicionales en el cálculo de la fórmula de Friedewald. Asimismo, Osorio et al. (2009) demuestran diferencia significativa entre el 
método directo y el método de precipitación en muestras de sangre equina para determinar valores de C-LDL. Este mismo grupo (Osorio y Uribe, 2011) no evidenciaron diferencias entre el método directo y la fórmula de Friedewald, por lo que no recomiendan el método de precipitación para tal fin. Nauck et al. (2002) afirman que la principal razón para seleccionar el método de referencia es tener exactitud y mantener la coherencia en las investigaciones y los resultados expuestos.

Los resultados no recomiendan el método de precipitación para la medición de colesterol LDL en el bovino, especie con patrón HDL. Los métodos sin duda merecen más estudios para establecer las aplicaciones más adecuadas en la cuantificación de C-LDL, ya que los resultados encontrados son difíciles de comparar, puesto que no existe mucha literatura como referencia en animales.

\section{Literatura Citada}

1. Aranda MV, Brave N, Casagrande R. 2012. Colesterol en bovinos. Sitio Argentino de Producción Animal. [Internet]. Disponible en: http:// www.produccionanimal.com.ar/ informacion_tecnica/carne_y_sub-productos/26-colesterol_en_bovinos.pdf

2. Bachorik PS, Ross $\bar{J} W$. 1995. National Cholesterol Education Program recommendations for measurements of low-density lipoprotein cholesterol: executive summary. National Cholesterol Education Program Working Group on Lipoprotein Measurements. Clin Chem 41: 1414-1420.

3. Bauchart D. 1993. Lipid absorption and transport in ruminants. J Dairy Sci 76: 3864-3881. doi: 10.3168/jds.S00220302(93)77728-0

4. Bauer JE. 1996. Comparative lipid and lipoprotein metabolism. Vet Clin Pathol 25: 49-56. doi: 10.1111/j.1939165X.1996.tb00968.x
5. Cirio A, Tebot I. 2000. Fisiología metabólica de los rumiantes. Montevideo, Uruguay: CSIC. 146 p.

6. Coppo JA. 1990. Effects of dietary lipidic charge in the concentration of bovine lipids and lipoproteins. Its influence on the saturation degree of fatty acids' storaged lipids. Acta Physiol Pharmacol Latinoam 40: 289-297.

7. Coppo JA. 1992. L'utilisation de suppléments nutritifs qui accroissent le degré de saturation des acides gras corporels des bovins. Ann Biol Clin 50: 263-264.

8. Coppo JA. 2001. Fisiología comparada del medio interno. Buenos Aires, Argentina: Ed Dunken. 315 p.

9. Fossati P, Prencipe L. 1982. Serum triglycerides determined colorimetrically with an enzyme that produces hydrogen peroxide. Clin Chem 28: 2077-2080.

10. Friedewald WT, Levy RI, Fredrickson DS. 1972. Estimation of the concentration of low-density lipoprotein cholesterol in plasma, without use of the preparative ultracentrifuge. Clin Chem 18: 499-502.

11. Mendes de Cordova CM, Schneider CR, Juttel LD, Mendes de Cordova M. 2004. Avaliacáo da dosagem directa do colesterol-LDL em amostras de sangue de 10.664 pacientes em comparacáo com o uso da fórmula de Friedewald. Arq Bras Cardiol 83: 482-487.

12. Montgomery R. 1998. Bioquímica: casos y texto. $6^{\mathrm{a}}$ ed. Madrid, España: Harcourt Brace. 681 p.

13. Nauck M, Warnick R, Rifai N. 2002. Methods for measurement of LDLcholesterol: a critical assessment of direct measurement by homogeneous assays versus calculation. Clin Chem 48: 236-254.

14. Osorio J, Castañeda J. 2018. Determinación de los niveles de colesterol LDL en ganado bovino comparando dos métodos. Rev Inv Vet Perú 29: xx-xx. doi: 10.15381/rivep.v29i1.14088. 
15. Osorio J, Uribe-Velásquez LF. 2011. Comparación de los métodos directo y de Friedewald para la determinación de los niveles de colesterol LDL en el equino. Rev MVZ Córdoba 16: 2549-2553.

16. Osorio JH, Barrera LM, Pérez JE. 2015. Comparación del perfil lipídico por sexo y edad en ovinos. Rev Med Vet Zoot 62: 11-19. doi: 10.15446/ rfmvz.v62n1.49381

17. Osorio JH, Carmona-Sepulveda J, Barrera-Ocampo LM. 2009. Comparación entre el método directo y el método de precipitación para la determinación de los niveles de colesterol HDL en equinos. Rev Colomb Cienc Pecu 22: 401-402.
18. Osorio JH, Vinazco J. 2010. El metabolismo lipídico bovino y su relación con la dieta, condición corporal, estado productivo y patologías asociadas. Biosalud 9(2): 56-66.

19. Valverde-Chaves G, Hidalgo-Quesada C, Echandi-Cruz L. 1994. Aplicabilidad de la fórmula de Friedewald y de un método de precipitación en la determinación del LDL colesterol. Rev Costarric Cienc 15: 43-50.

20. Warnick GR, Knopp RH, Fitzpatrick V, Branson L. 1990. Estimating lowdensity lipoprotein cholesterol by the Friedewald equation is adequate for classifying patients on the basis of nationally recommended cut points. Clin Chem 36: 15-19. 